\title{
Deterministic Optical Rogue Waves
}

\author{
Cristian Bonatto, ${ }^{1}$ Michael Feyereisen, ${ }^{2}$ Stéphane Barland, ${ }^{2}$ Massimo Giudici, ${ }^{2}$ Cristina Masoller, ${ }^{1}$ \\ José R. Rios Leite, ${ }^{2,3}$ and Jorge R. Tredicce ${ }^{2,3}$ \\ ${ }^{1}$ Departament de Fìsica i Enginyeria Nuclear, Universitat Politècnica de Catalunya, Colom 11, E-08222 Terrassa, Barcelona, Spain \\ ${ }^{2}$ Université de Nice Sophia Antipolis, Institut Non-Linéaire de Nice, UMR 6618, F-06560 Valbonne, France \\ ${ }^{3}$ Departamento de Fisica, Universidade Federal de Pernambuco, 50670-901, Recife, Brazil
}

(Received 17 May 2011; published 29 July 2011)

\begin{abstract}
Experimental observations of rare giant pulses or rogue waves were done in the output intensity of an optically injected semiconductor laser. The long-tailed probability distribution function of the pulse amplitude displays clear non-Gaussian features that confirm the rogue wave character of the intensity pulsations. Simulations of a simple rate equation model show good qualitative agreement with the experiments and provide a framework for understanding the observed extreme amplitude events as the result of a deterministic nonlinear process.
\end{abstract}

DOI: 10.1103/PhysRevLett.107.053901

PACS numbers: 42.65.Sf, 05.45.-a, 42.60.Mi, 42.55.Px

According to fishermen tales from a pub in Ireland, rogue waves (RWs) like solid walls of water, higher than $30 \mathrm{~m}$, are more or less common phenomena in deep ocean waters. This fact is in contradiction with the Gaussian models often used to describe fluctuations of the wave height in the sea [1,2]. A recent experience of a luxury ship close to Antarctica is an example that seems to give credit to such tales. Scientific interest on extremely high waves increased substantially during the past decade not only in oceanography but also in other systems such as capillary waves [3], acoustic waves [4], and optical waves [5-10]. Both from the theoretical and from the experimental points of view there are several issues still unclear, such as the physical mechanisms that originate the RWs, the way they develop [11], the probability to observe them [12], and the type of system able to generate such extreme events [13].

A first problem is defining quantitatively an extreme event. The oceanography community often employs the abnormality index, which is the ratio between the height of the wave and the average wave height among one-third of the highest waves in a time series [14]. Every event whose abnormality index is larger than 2 is considered a rogue wave. An alternative definition is in terms of the standard deviation $\sigma$ of the ocean surface variations: any wave whose height is higher than the mean surface value plus $8 \sigma$ is considered a rogue wave [14].

These definitions have the advantage of being precise and the drawback of being quite arbitrary. Since they imply that a rogue wave is highly improbable if the probability distribution function (PDF) of the wave amplitude is a Gaussian, the optical community has employed the general criterion that non-Gaussian and long-tailed PDFs signature the existence of RWs [5-10].

A second problem is determining which type of system might exhibit these rare extreme events. The intrinsic characteristics of a rogue wave (high amplitude, fast rise and fast fall) imply that the system must be highly nonlinear. A mechanism that has been shown to be directly connected to RWs is the existence of a modulational instability [15], as in the nonlinear Schrödinger (NLS) equation. From the theoretical point of view, the formation of ocean rogue waves has been studied using as a framework the NLS equation (see, e.g., Refs. [16,17] and references therein) in spite of that fact this equation cannot explain by itself ocean waves in short scales [15]. It is therefore not surprising that other systems also modeled by the NLS equation, such as mode-locked lasers and Kerr media, may display rogue waves.

An important open problem is the role of noise. One can ask if rogue waves can be described by a fully deterministic process, with noise acting as a driving force increasing or decreasing the probability of observing RWs. Related to this is determining which are the minimum nonlinear ingredients required for an increase in the probability of RWs. These are the main objectives of the present work together with the demonstration that optical RWs appear in a simple (and relatively inexpensive) experimental setup: a commercial semiconductor laser subjected to $\mathrm{cw}$ optical injection from a master laser. In spite of the fact that optically injected semiconductor lasers have been extensively studied (not only because of their many applications, but also because of their rich variety of dynamical behaviors), the fact that they can generate such giant pulses has not yet been reported.

Here we characterize experimentally the parameter region where rogue waves appear and present results of simulations of a simple model that are in good qualitative agreement with the experimental observations.

In the experiments the light emitted by a tunable continuous-wave master laser is injected into a slave laser whose pump current and temperature are stabilized better than $0.1 \mu \mathrm{A}$ and $0.01 \mathrm{~K}$. The master laser can be tuned in the range $960-980 \mathrm{~nm}$ and its linewidth is less 
than $1 \mathrm{MHz}$ on long time acquisitions (larger than $1 \mathrm{~min}$ ). The slave laser is a $980 \mathrm{~nm}$ vertical cavity surface emitting laser (VCSEL) with threshold at about $0.2 \mathrm{~mA}$ that emits on the fundamental transverse mode up to $2.8 \mathrm{~mA}$. The experiments were done keeping the slave laser current well below the limit of single transverse mode emission. Within this current range the slave laser also emits a constant polarization. The power of the injected light (whose polarization is aligned to that of the slave laser) can be adjusted up to $2 \mathrm{~mW}$. The slave laser output passes through an optical isolator which avoids backreflection from the detection part of the setup. This consists of a $9.5 \mathrm{GHz}$ photodetector whose output is monitored on a $6 \mathrm{GHz}$ oscilloscope (LeCroy Wavemaster) after passing in a $30 \mathrm{~dB}$ amplifier (10 MHz-4.2 GHz). The optical spectra are detected with a scanning Fabry-Perot interferometer (free spectral range of $78 \mathrm{GHz}$ and finesse of 125).

In an optically injected laser the frequency detuning between the master and the slave lasers, $\Delta \nu=\nu_{o}-\nu_{s}$, the injection intensity, $P_{\text {inj }}$, and the slave laser pumping current, $J$, are crucial parameters. However, they are not independent as the emission frequency of a VCSEL depends sensibly on its pump current due to Joule heating. For the device used in the experiments the variation was of about $116 \mathrm{MHz} / \mu \mathrm{A}$ at $J=1 \mathrm{~mA}$. In Fig. 1(a), which displays the bifurcation diagram obtained by measuring the slave laser optical spectrum versus $J$, when $J$ changes from 0.867 to $1.046 \mathrm{~mA}, \Delta \nu$ also varies between -14 and $+7 \mathrm{GHz}$, with $\Delta \nu=0$ occurring at $J=0.987 \mathrm{~mA}$ (indicated with a dashed line).
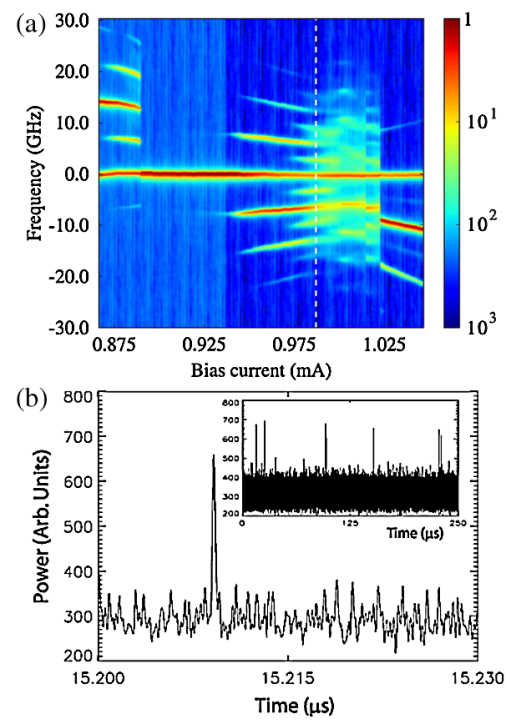

FIG. 1 (color online). (a) Experimental bifurcation diagram displaying the optical spectra of the slave laser versus the bias current. The dashed line denotes the zero detuning condition between the master and the slave lasers. (b) Typical time series of the slave laser intensity, where rare large pulses can be observed. The experimental parameters are $J=0.976 \mathrm{~mA}$, $\Delta \nu=-1.34 \mathrm{GHz}$, and $P_{\text {inj }}=1.1 \mathrm{~mW}$.
In Fig. 1(a) we can distinguish four regions: for large and negative detuning there are beating oscillations; as the pump current increases (and $\Delta \nu$ decreases), a first bifurcation originates a period two of the beat note, then frequency pulling leads to a locking region that is characterized by the absence of oscillations in the slave laser and persists over a wide range of pump currents. A further increase of the slave laser pump current leads to the end of the locking region, which begins with periodic oscillations and ends up in a chaotic behavior after a period doubling sequence. For $J>1.025 \mathrm{~mA}$, there is no interaction between the slave and master lasers and their emission lines become independent.

Figure 1(b) displays a typical time series of the slave laser intensity within the third region. Close to the border but inside the chaotic region, the intensity shows a series of small pulses with sporadic extremely high ones. We interpret these rare events as rogue waves. To confirm their RW character, histograms of the laser peak intensity were done for different values of the slave laser pump current, to uncover the parameter region where the associated PDF displays a long tail, typical of rogue waves. The histogram displayed in Fig. 2(a) clearly indicates that rogue waves were not present in the time series. However, for higher values of the slave laser current the probability of high pulses increases, and the corresponding histogram, displayed in Fig. 2(b), indeed has a long tail that reveals the existence of RWs. For even larger pump current values, the probability of high intensity peaks grows and the high peaks gradually lose their RW character, as RWs, by definition, are rare events.

Notice that in Fig. 2(b) there is a range of power values where no RW events occur. This is probably due to the finite length of the time series and, therefore, to the small number of RW events, which are indeed very rare. However, we cannot exclude the existence of physical mechanisms that result in a nearly forbidden "gap" region of pulse amplitude values in phase space.

This bifurcation scenario and, more specifically, the presence of these sporadic high intensity pulses is found
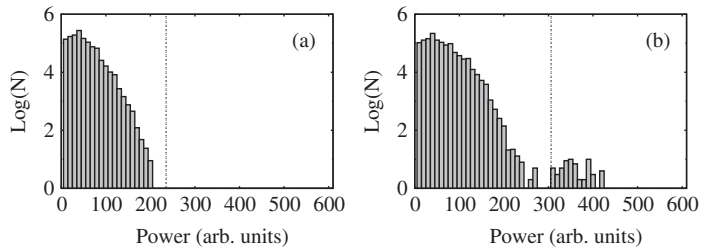

FIG. 2. Experimental histograms, without (a) and with (b) rogue waves. $N$ is the number of pulses detected on the time traces of the slave laser intensity. The vertical dashed lines denote the mean value plus 8 standard deviations and thus mark the limit for a pulse to be considered a rogue wave. The histogram in (b) corresponds to the time series displayed in Fig. 1(b) $(J=0.976 \mathrm{~mA})$, while the histogram in (a) was obtained by slightly decreasing the current $(J=0.972 \mathrm{~mA})$. 
in a wide range of optically injected power (from 0.6 to $1.4 \mathrm{~mW}$ ). We observed that the critical detuning for which these events occur approaches zero as $P_{\text {inj }}$ decreases and can also be positive for low injected power. The range of injected power where RWs are observed depends also on the slave laser pumping current. If $J$ is increased to $1.321 \mathrm{~mA}$ this range is shifted upwards (from 1.0 to $2.0 \mathrm{~mW}$ ) while the critical detuning remains almost unchanged. We also remark that the occurrence of rogue events disappears for $J>2 \mathrm{~mA}$ as well as the entire third region of the bifurcation diagram; upon increasing the bias current, the slave laser unlocks suddenly becoming independent of the master laser.

The experimental observations can be well understood in terms of the usual rate equations for a semiconductor laser with cw optical injection:

$$
\begin{gathered}
\dot{E}=\kappa(1+i \alpha)(N-1) E+i \Delta \omega E+\sqrt{P_{\text {inj }}}+\sqrt{D} \xi, \\
\dot{N}=\gamma_{n}\left(\mu-N-N|E|^{2}\right),
\end{gathered}
$$

where $E$ and $N$ are the slowly varying complex amplitude and the carrier density of the slave laser, respectively, $P_{\text {inj }}$ is the optical injection strength, $\Delta \omega=\omega_{s}-\omega_{o}$ is the angular frequency detuning between the master and the slave lasers, $D$ is the noise strength, and $\xi$ is a complex Gaussian white noise representing spontaneous emission. Other parameters are the field decay rate, $\kappa$, the carrier decay rate $\gamma_{n}$, the linewidth enhancement factor $\alpha$, and the injection current parameter $\mu$.

To investigate the parameter region where rogue waves can be found, we first compute the Lyapunov exponents of the deterministic model [18] as a function of two of the experimental parameters: the frequency detuning, $\Delta \nu=$ $\Delta \omega / 2 \pi$, and the pump current of the slave laser, $\mu$. One should notice that in the model a change in $\mu$ does not imply a change in $\Delta \nu$; therefore, the experimental situation corresponds, in the simulations, to changing both parameters simultaneously.

The results of the calculation of the Lyapunov exponents are displayed in Fig. 3(a), where the bright colors indicate regions of deterministic chaotic behavior.

Changing $\mu$ and $\Delta \nu$ along the indicated line results in a sequence of bifurcations that is very similar to that observed experimentally: for low $\mu$ the laser displays periodic oscillations (beating). Increasing $\mu$, the laser enters the locking region. With further increase of $\mu$ the laser unlocks and displays periodic oscillations which are followed by a period doubling sequence to chaos. With even further increase of $\mu$, the chaotic region ends abruptly and the laser again displays periodic oscillations.

In the deterministic, noise-free equations, RWs were found for certain parameter values within the chaotic region. Figure 3(b) displays in detail the parameter region
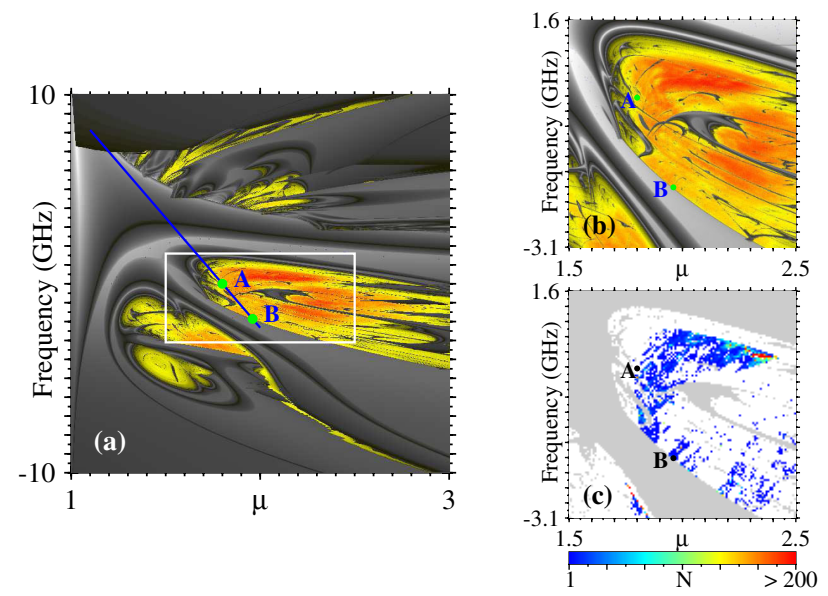

FIG. 3 (color online). (a) Lyapunov exponents as function of slave laser pump current and the master-slave frequency detuning. The color code indicates negative exponents as gray scale and positive exponents as black-white (yellow-red) scale. The solid line indicates a simultaneous variation of $\mu$ and $\Delta \nu$ that results in a sequence of bifurcations very similar to that observed experimentally. (b) Detail of box shown in (a). The points labeled $A$ and $B$ indicate the parameters corresponding to Fig. 4. (c) Number of RWs, $N$, vs the slave laser pump current and the frequency detuning. $N$ was calculated over time series spanning $10 \mu \mathrm{s}$. The model parameters are $\alpha=3, \kappa=$ $300 \mathrm{~ns}^{-1}, \gamma_{n}=1 \mathrm{~ns}^{-1}, P_{\text {inj }}=60 \mathrm{~ns}^{-2}$, and $D=0$.

where they occur. Within the chaotic region, a typical time trace of the laser intensity, without RWs, is displayed in Fig. 4(a), where one can observe irregular oscillations. This time trace corresponds to point $A$ in Fig. 3(b). In point $B$ (for larger $\mu$ and smaller $\Delta \nu$ ), the intensity displays sporadic very high pulses, an example of one of them is shown in Fig. 4(d). These pulses can be identified
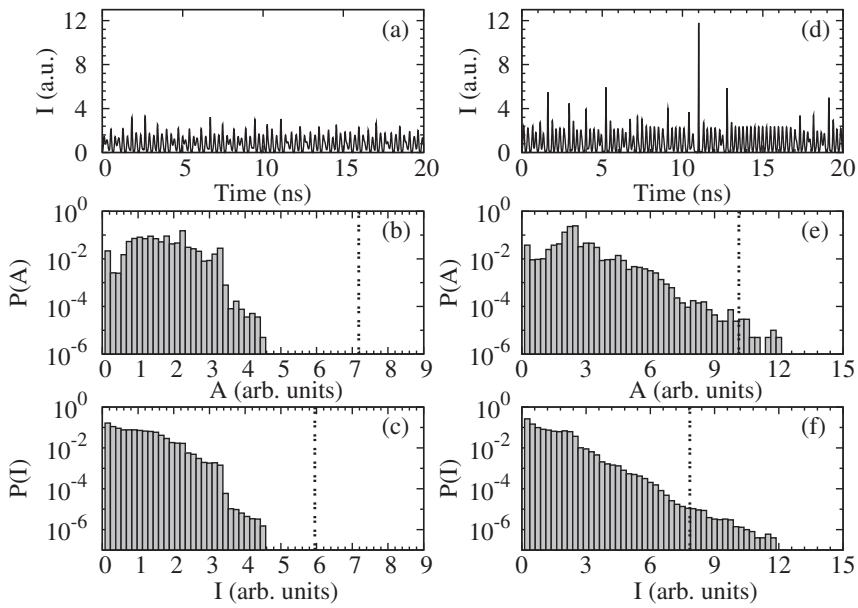

FIG. 4. Numerically calculated laser intensity (a),(d) and the PDFs of the pulse amplitude (b),(e) and intensity (c),(f), for parameters corresponding to point $A$ (a)-(c) in Fig. 3 ( $\mu=1.8$, $\Delta \nu=0 \mathrm{GHz})$, and to point $B(\mathrm{~d})-(\mathrm{f})$ in Fig. $3(\mu=1.96, \Delta \nu=$ $-1.86 \mathrm{GHz})$. 

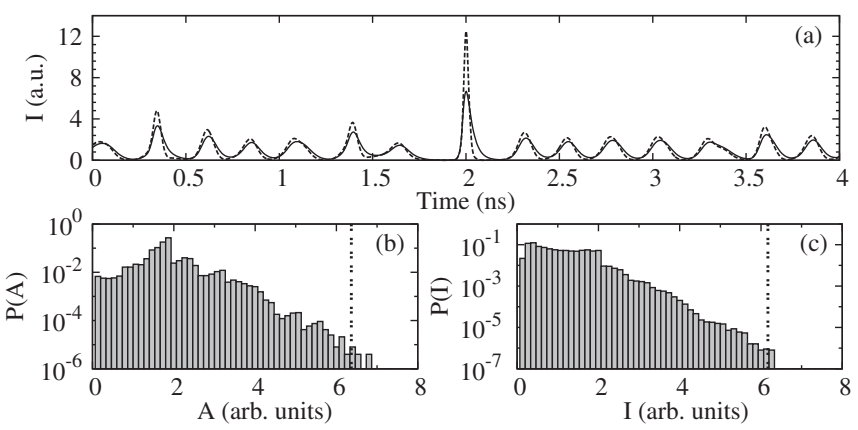

FIG. 5. (a) Time series of the laser intensity obtained by simulating the stochastic rate equations, without filter (dashed line) and with filter (solid line). PDFs of the pulse amplitude (b) and intensity (c). The noise strength is $D=10^{-5} \mathrm{~ns}^{-1}$; other parameters are as in Fig. 4, right-hand column.

as RWs, as the PDFs of the amplitude of the intensity pulses, $P(A)$ [Fig. 4(e)], and also of the laser intensity, $P(I)$ [Fig. 4(f)], are long-tailed. In contrast, for the time series without high pulses, the corresponding PDFs [Figs. 4(b) and 4(c)] display an abrupt cutoff.

The precise definitions of RWs previously discussed can also be employed, and a high intensity pulse can be identified as a RW by using any of these two criteria: (a) if it is higher than $\langle A\rangle+8 \sigma_{A}$, and (b) if it is higher than $\langle I\rangle+8 \sigma_{I}$, where $\langle I\rangle$ and $\langle A\rangle$ are the average intensity and average pulse amplitude, and $\sigma_{I}$ and $\sigma_{A}$ are the corresponding standard deviations. As can be seen in the PDFs shown in Fig. 4, the these two criteria define two borders, and there are several pulses that can be identified as RWs.

The number of such RW events is plotted in Fig. 3(c) as a function of the pump current parameter and frequency detuning [in this figure the rogue waves were identified by using the criterion (a)]. By comparing Figs. 3(b) and 3(c) one can see that the RWs occur in subregions of the chaotic region.

It is important to remark that the above presented results were obtained from simulations of the deterministic model, and that they are in good qualitative agreement with the experimental observations, which suggests that, in this model and for the parameters explored, the rogue waves originate from deterministic nonlinearities.

Since an amplifier was employed in the detection part of the experimental setup (which results in a filtering process of the data), and there is also the unavoidable presence of noise, let us now include these features in the model and investigate their influence. Figure 5 displays results of simulations with a realistic value for the noise strength $D$ and a low pass filter (cutoff frequency at $5 \mathrm{GHz}$ ). The filter affects mainly the high amplitude intensity pulses, which are considerably reduced as compared with the unfiltered time series, as shown in Fig. 5(a). In this way, the main effect of the filter is to reduce the number of pulses that satisfy the rogue wave criteria. The PDFs do not change significantly when noise and filter are included, as shown in Figs. 5(b) and 5(c), confirming that the determinism and nonlinearities present in the theoretical description are the key ingredients for originating the rogue waves in the system.

In conclusion, we studied experimentally and theoretically the dynamics of an optically injected semiconductor laser and identified giant intensity pulses as RWs. Histograms of pulse height were systematically measured for parameters where the laser emits chaotically, and in some regions long-tailed PDFs were observed. Simulations based on a simple noise-free rate equation model gave results in good qualitative agreement with the experiments, allowing for the interpretation of the sporadic high amplitude pulses as the result of a deterministic nonlinear process. The addition of realistic spontaneous emission noise did not change the above presented results, at least in the parameter region explored. However, the interplay of nonlinearity and noise can result in nontrivial effects, which can in turn inhibit or trigger RWs, and a detailed investigation is left for future work.

As the rogue wave phenomenon is relevant not only for nonlinear physics and applied engineering but also even for economic sciences [19], we expect that our results will have a large impact in the community, as we have (1) provided a simple and inexpensive setup for the experimental study of optical RWs and (2) demonstrated that they can originate from fully deterministic nonlinear processes.

The authors acknowledge partial support from EOARD Grant No. FA-8655-10-1-3075 and Brazilian projects Capes-Cofecub-456 and CNPQ-Facepe-Pronex APQ0630-1.05/06. C. M. also acknowledges support from the ICREA Academia program.

[1] M. S. Longuet-Higgins, Phil. Trans. R. Soc. A 249, 321 (1957).

[2] S. Aberg and G. Lindgren, Prob. Eng. Mech. 23, 359 (2008).

[3] M. Shats, H. Punzmann, and H. Xia, Phys. Rev. Lett. 104, 104503 (2010).

[4] A. N. Ganshin et al., Phys. Rev. Lett. 101, 065303 (2008).

[5] D. R. Solli et al., Nature (London) 450, 1054 (2007).

[6] J. M. Dudley, G. Genty, and B. J. Eggleton, Opt. Express 16, 3644 (2008).

[7] A. Mussot et al., Opt. Express 17, 17010 (2009).

[8] M. Kovalsky, A. A. Hnilo, and J. R. Tredicce (to be published).

[9] A. Montina et al., Phys. Rev. Lett. 103, 173901 (2009).

[10] F. T. Arecchi et al., Phys. Rev. Lett. 106, 153901 (2011).

[11] C. Kharif and E. Pelinovsky, Eur. J. Mech. B, Fluids 22, 603 (2003).

[12] B. S. White and B. Fornberg, J. Fluid Mech. 355, 113 (1998).

[13] K. Hammani et al., Phys. Lett. A 374, 3585 (2010).

[14] C. Kharif, E. Pelinovsky, and A. Slunyaev, Rogue Waves in the Ocean (Springer, Heidelberg, 2009). 
[15] A. I. Dyachenko and V. E. Zakharov, JETP Lett. 81, 255 (2005).

[16] A. R. Osborne, M. Onorato, and M. Serio, Phys. Lett. A 275, 386 (2000).
[17] B. Kibler et al., Nature Phys. 6, 790 (2010).

[18] C. Bonatto, J. C. Garreau, and J. A. C. Gallas, Phys. Rev. Lett. 95, 143905 (2005)

[19] Z. Y. Yan, Commun. Theor. Phys. 54, 947 (2010). 